\title{
Caregiving tasks and caregiver burden; effects of an psycho-educational intervention in partners of patients with post-operative heart failure
}

\author{
Susanna Ågren, Anna Strömberg, Tiny Jaarsma and Marie Louise A. Luttik
}

\author{
Linköping University Post Print
}

\section{Tweet}

N.B.: When citing this work, cite the original article.

Original Publication:

Susanna Ågren, Anna Strömberg, Tiny Jaarsma and Marie Louise A. Luttik, Caregiving tasks and caregiver burden; effects of an psycho-educational intervention in partners of patients with post-operative heart failure, 2015, Heart \& Lung, (44), 4, 270-275.

http://dx.doi.org/10.1016/j.hrtlng.2015.04.003

Copyright: Elsevier http://www.elsevier.com/

Postprint available at: Linköping University Electronic Press http://urn.kb.se/resolve?urn=urn:nbn:se:liu:diva-120214 


\section{Abstract \\ Objective}

To evaluate the effects of a psycho-educational intervention on perceived caregiver burden in partners of patients with postoperative heart failure.

\section{Background}

Since partners of cardiac surgery patients play a significant role in the patient's recovery, it is important to also address their needs during hospitalization and after discharge.

\section{Methods}

Forty-two patients with postoperative heart failure and their partners participated in a randomized controlled pilot study. Dyads in the intervention group received psychoeducational support from a multidisciplinary team. Dyads in the control group received usual care.

\section{Results}

No significant differences were found in the performance of caregiving tasks and perceived caregiver burden in the control versus the intervention group.

\section{Conclusion}

A pilot study exploring the effects of a psycho-educational intervention in patients and their partners did not reveal significant effects with regard to reduced feelings of burden in partners. Alleviating caregiver burden in partners may need a more intense or specific approach. More large scale intervention studies are necessary to gain robust knowledge on effective intervention strategies to support partners and patients with postoperative heart failure. 


\section{Introduction}

Cardiac surgery is extremely stressful for patients and their partners. ${ }^{1,2}$ The complication of heart failure after cardiac surgery may have an even greater influence on both the patient's and their partner's everyday life. ${ }^{1-3}$ The consequences of cardiac disease and cardiac surgery are known for their impact on patient's and their spouse's quality of life. ${ }^{4}$ Patients and their partners are confronted with postoperative burden in terms of impaired emotional and functional well-being and as a result they often struggle with feelings of anxiety, depression and impaired quality of life. ${ }^{5,6}$ Additionally, patients with PHF and their partners are confronted with a complex, often chronic treatment regimen, which is an unexpected result of their cardiac surgery.

Surgical (and percutaneous) coronary revascularization is indicated in patients with severe coronary artery disease $(\mathrm{CAD})$ and in patients with heart failure for the relief of angina pectoris. ${ }^{7}$ Heart failure can be defined as 'a hemodynamic state secondary to pump failure unable to meet systemic demands without treatment other than correction of volume or vascular resistance'. ${ }^{7}$ In the perioperative phase of cardiac surgery, heart failure (HF) is one of the most common conditions requiring evaluation and treatment. The incidence of developing heart failure after cardiac surgery among patients with existing cardiac conditions, such as coronary heart disease, prior heart failure, or valvular heart disease, is approximately $10 \% .8$ Postoperative heart failure (PHF) is a serious complication that remains the main cause for early mortality after cardiac surgery. It is also associated with increased postoperative morbidity and serious secondary complications..$^{8-10}$

Partners of patients play a vital role in the patient's recovery after cardiac surgery. ${ }^{2}$ Support from partners of patients with chronic heart failure has proven to be beneficial with regard to the patient's quality of life and survival ${ }^{11,12}$ but also in terms of patient self-care ${ }^{13}$ and patient adherence to therapy. ${ }^{14}$ However, burdensome caregiving can cause impaired 
mental and physical health and even untimely death in partners or other caregivers. ${ }^{15,16}$ Ågren et $\mathrm{al}^{6}$ specifically addressed the needs and experiences of spouses of patients with complications of HF after cardiac surgery. The study revealed that in order to be able to cope with the stressful situation, spouses needed to feel acknowledged and involved as being an important person ${ }^{6}$.

Caregiver burden can be analyzed by making a distinction between two different aspects; the performance of caregiving tasks and perceived feelings of caregiver burden. ${ }^{17,18}$ Partners of patients with chronic heart failure mainly perform tasks with regard to practical and emotional support. ${ }^{19}$ Perceived caregiver burden refers to the caregiver's reaction or appraisal of their caregiving role. ${ }^{17,18,20}$ Both aspects of caregiver burden are relevant and may need different kind of interventions. Although some studies have described limited associations between the performance of caregiving tasks and feelings of caregiver burden in patients with $\mathrm{HF}^{19,20}$, and in caregivers of dementia patients ${ }^{21}$, not much is known regarding the relationship between these two aspects in caregivers of patients with postoperative HF.

Psychosocial support interventions for patients and their families can be categorized into (1) interventions that are focused on educating patients and their family members about the disease and its treatment, (2) interventions that are focused on helping families cope more effectively with the disease by teaching problem-solving skills, and (3) interventions that may be appropriate in families that are highly dysfunctional, independent of the family member's chronic disease. ${ }^{22}$ A meta- analysis of psychosocial family interventions indicated that studies that involved the partner positively affected the patients' health and the partners' health in terms of decreased caregiving burden, depression, and anxiety ${ }^{23}$. There was a tendency towards higher effects in relationship-oriented, psycho-educational family interventions, compared to educational interventions ${ }^{23,24}$. 
Having outlined several challenges for dyads of HF patients and what is known from earlier research, a supportive, psycho-educational intervention addressing patients and their partners was designed. ${ }^{25}$ The aims of the present study are twofold; firstly to evaluate the effects of a psycho-educational intervention to patients with PHF and perceived caregiver burden in their partners. Secondly, the aim is to explore the association between two aspects, of caregiver burden in partners of patients with PHF, namely performed caregiving tasks and perceived burden

Research questions;

1. What are the effects of a psycho-educational intervention on perceived burden in partners of patients with postoperative heart failure?

2. Are there associations between the performance of caregiving tasks and perceived caregiver burden in partners of patients with postoperative heart failure?

\section{Method}

Design

A randomized controlled pilot study comparing an intervention group receiving a psychoeducational to a control group receiving care as usual was used to explore the effects of this intervention in patients with PHF.

\section{Participants and recruitment}

Longitudinal data of a pilot intervention study in partners of patients with PHF at a university hospital in south-east Sweden was used. Other findings from this study (on patients` and partners' health, quality of life and perceived control) have been published elsewhere. ${ }^{25}$ In this study PHF was defined as; having a clinical diagnosis of HF determined by the attending physician, receiving treatment for PHF, such as a circulatory assist device or inotropic support 
for $\geq 24 \mathrm{~h}$ postoperatively, and a postoperative stay in the intensive care unit for $\geq 48 \mathrm{~h}$. To be eligible for the study patients had to meet all three criteria. Inclusion criteria were that the patient lived with a partner and that the dyad understood written and spoken Swedish. Exclusion criteria were a short life expectancy due to a life threatening disease other than heart failure (e.g. cancer) or impaired cognitive ability as documented in the medical record. According to the rule of thumb for pilot trial studies ${ }^{26}$ a sample of 12 per group was used. However, to ensure that an appropriate number of dyads will receive the complete intervention, a sample size of 15-20 dyads was pursued. Patients and partners were randomized to either the control condition or the experimental condition by using blocks. The Regional Ethical Review Board in Linköping (Dnr M178-04) approved the study, and the principles outlined in the Declaration of Helsinki were followed. An information letter was sent out to patients and their partners within two weeks after discharge. A research nurse then called patients and partners in order to provide additional verbal information. Patients and their partners were asked to participate and return their written informed consent if they agreed to take part.

\section{Control condition}

Patients and their partners routinely received standard care by members of a cardiac surgery care team. The patients received information from the thoracic surgeon, thoracic anesthetist and a nurse during hospital admission before surgery. One week after discharge, the same nurse contacted the patients for a follow-up call. Patients then received non-structured information about recovery, medication, rehabilitation, and follow-up procedures. Partners of patients were not systematically involved in the provision of information. 


\section{Experimental condition}

In addition to standard care, the patients and their partners in the intervention group received psycho-educational support at 2-4 weeks after discharge from a multidisciplinary team consisting of a physician, a nurse, and a physiotherapist. During these meetings, each professional used a semi-structured dialogue guide. Within these sessions, patients and partners were invited to share their experiences, discuss situations that caused stress, and also to talk about possible effects on their relationship and their mutual need for support. Dyads who experienced psychosocial distress that was difficult to handle, could be referred to a social worker.

The intervention consisted of 3 sessions lasting for 30-60 minutes. The first session was planned at 4-6 weeks after discharge; and the second and third session were scheduled at 1012 weeks and 22-24 weeks after discharge, respectively. During the first session patients and their partners visited the outpatient clinic where they met all the members of the care team. During the second and third session the nurse provided education and support through telephone consultation. Separate conversations were held with the patient and the partner. The intervention was delivered according to a pre-specified intervention protocol. The use of semi-structured dialogue guides ensured the content and consistency of the intervention delivery. The content of the conversations and discussions was summarized in writing after each session, and these were used to connect different sessions.

\section{Data collection}

Data was collected between May 2007 and August 2011. Information letters and questionnaires were sent by post at baseline (within 2 weeks of discharge). The second questionnaire was completed at 3 months after the patient's discharge and shortly before the second psycho-educational support session. At 12 months after discharge the final 
questionnaires were sent to determine long-term effects of the psycho-educational support sessions. Questionnaires were returned in a prepaid envelope. This paper only reports on outcomes related to caregiver burden, other patient and partner outcomes have been reported elsewhere $^{25}$

\section{Measures}

Demographic and background data was collected using a self-administered questionnaire identifying age, gender, education and work situation.

To measure perceived caregiver burden, the Caregiver Burden Scale (CBS) developed by Elmsthåhl and colleagues was used. ${ }^{27}$ The CBS consists of 22 items using a 4-point Likert scale format, measuring five dimensions of caregiver burden; general strain, disappointment, isolation, emotional involvement and environment. Each dimension has a theoretical score between 1 and 4, with a high score indicating a high level of perceived burden. The total burden index CBS score can be divided into low burden (1.00-1.99), medium burden (2.002.99), and high burden (3.00-4.00). The CBS was found to be a valid and reliable scale to assess caregiver burden in partners of patients with stroke and dementia. ${ }^{27}$ Internal consistency in the current data set varied, with the lowest Cronbach's Alpha (0.74) for the subscale environment and the highest (0.93) for the total scale.

The Dutch Objective Burden Inventory (DOBI), developed by Luttik et al. ${ }^{18}$ assesses caregiving tasks performed by HF partners. The DOBI is a self-administered questionnaire containing 38 items (or tasks) using a 3-point Likert scale format. The DOBI can be divided into four domains; personal care, practical care, motivational support, and emotional support. Scores per domain range from 1 to 3, with a high score indicating a high burden. Different studies have shown adequate internal consistency (Cronbach's alpha > .80) and construct 
validity for this instrument. ${ }^{18,28}$ Cronbach's Alpha in the current data varied from 0.88 for the subscales practical and motivational support to 0.94 for the subscale emotional support.

\section{Data analyses}

Descriptive statistics were used to describe the study population. Analysis of frequencies was performed using the Chi-square or Mann Whitney U test for the categorical variables; the Student's t-test was used to compare groups with regard to age. The Mann Whitney U (twotailed) test was used for differences between the control and intervention group. In order to analyze trends in caregiver burden over time, difference scores between baseline and 3 months (baseline score - 3 months score), and baseline and 12 months (baseline score -12 months score) were calculated for each individual (partner). The Mann Whitney U (twotailed) test was used for testing differences in the mean difference score between groups. The (two-tailed) Spearmans'rho correlation coefficient was used to analyze and describe associations between the performance of caregiving tasks and perceived caregiver burden. Statistical analyses were performed using SPSS 20.0 for Windows. An overall significance level of $\mathrm{p}<0.05$ was used

\section{Results}

\section{Study population}

In total 50 dyads were eligible for participation in the study. However, 8 dyads declined participation due to conditions such as fatigue and stress and therefore, 42 dyads completed baseline assessment. Seventeen dyads were randomized to the control group, and 25 patients were randomized to the intervention group. 
Patients in the intervention group had a mean age of $69( \pm 8)$ years, whereas patients in the control group had a mean age of $70( \pm 9)$ years. Patients were predominantly male $(88 \%)$.Comorbidities such as diabetes, COPD and stroke were present in both groups (Table 1.) According to the calculated EuroSCORE, patients were generally at high-risk, with a trend $(p=0.08)$ towards a higher EuroSCORE in the control group $(9.8 \pm 4.3)$ compared to the intervention group $(7.8 \pm 3.2)$. Ten patients had died after 12 months, 4 in the control group and 6 in the intervention group. Patient's mean length of hospitalization was $16( \pm 11)$ days, of which 4.1 days (mean) were spent in intensive care due to complications such as postoperative heart failure.

The mean age of partners was $67( \pm 7)$ in the intervention group, and $66( \pm 8)$ years in the control group. Eighty-four percent of the partners were female in the intervention group, whereas there were $94 \%$ women in the control group. Partners in the intervention group had a significantly higher educational level (Table 1).

\section{Effects of the intervention}

In total 19 dyads completed all three phases of the intervention and therefore received the complete intervention. Attrition of dyads was fully due to six patients who had died during the first months of follow-up. Caregiver burden scores ranged between 1.2 and 1.9 on the theoretical range of 1-5. Sixty-nine percent of partners had a total CBS index score below 1.99, 29\% had a medium score between 2.00 and 2.99, and 1 partner $(2 \%)$ had a high burden with a score above 3.00. DOBI scores ranged between 1.06 on support in personal care and 2.00 on emotional support within a theoretical range between 1 and 3 . Table 2 shows the mean scores on the CBS and DOBI subscales for partners in the control group and the intervention group. Analyses of these DOBI and CBS scores did not show any significant 
differences ( $\mathrm{p}$ varying from 0.08 to 0.99 ) in caregiver burden between the two treatment conditions (Table 2).

\section{Changes in caregiver burden over time}

Difference scores were calculated to explore the development of perceived caregiver burden over time in both the control group and the intervention group. The data indicate that almost all scores on the DOBI subscales decreased during the year after discharge compared to baseline scores (expressed in positive difference scores in Table 3). The decrease in burden scores at 3 and 12 months seems most prominent for the subscales personal care and emotional support in the intervention group. However, differences in change over time between the control group and the intervention group did not reach significance, with p-values varying from 0.06 (emotional support) to 0.93 (motivational support). Scores on the CBS for perceived caregiver burden showed the most prominent decrease at three months. No significant differences in change over time were found between the control group and the intervention group, with p-values varying from 0.05 (environmental) to 0.99 (total score and general strain).

At 3 months, 9 patients had died. We compared the burden measured by DOBI and CBS and there were no significant differences in aspects of caregiver burden at baseline between the partners of patient who had died and those who completed the study.

\section{Association between performance of tasks and perceived burden}

At baseline some significant associations were found between performing tasks and perceived caregiver burden. Performing practical tasks was moderately associated with the total CBS score $(\mathrm{rho}=0.31, \mathrm{p}<0.05)$, general strain $(\mathrm{rho}=0.39, \mathrm{p}<0.05)$ and feelings of isolation 
$(\mathrm{rho}=0.35, \mathrm{p}<0.05)$. Motivational support was significantly associated with most of the CBS subscales, rho ranging from 0.41 to $0.60, \mathrm{p}<0.01$ (Table 5).

At 12 months, associations between performing caregiving tasks and perceived caregiver burden were more prominent. All DOBI subscales were significantly associated with the CBS total score, general strain, isolation, and disappointment, with rho's ranging from 0.39 $(\mathrm{p}<0.05)$ to $0.58(\mathrm{p}<0.01)$.

At baseline as well as at 12 months, the associations between performing caregiving tasks and the CBS subscales emotional involvement and environment appeared smaller.

\section{Discussion}

A dyad intervention consisting of 3 psycho-educational sessions from a multidisciplinary healthcare team to PHF patients and their partners after cardiac surgery did not reveal significant effects with regard to reduced caregiver burden in partners. The performance of caregiving tasks and perceived caregiver burden decreased at 3 months in both the control and the intervention group. No significant differences in changes over time between the groups were found.

There may be various explanations why no significant effects of this intervention on the partners' caregiver burden were found. First of all, the study was performed as a randomized pilot study with a small sample size of 42 dyads. Due to this small sample size the study was underpowered to reach statistical significance. Secondly, levels of burden were moderate to low at baseline in both groups. Sixty-nine percent of the partners scored low $(<1.99)$ on the total burden index score of the CBS. These findings are comparable to studies by Luttik et al. ${ }^{19}$, Makdessie et al. ${ }^{28}$ and Ågren and collegues ${ }^{29}$, in which a considerable number of partners did not report feeling burdened. The time chosen for the baseline measurement (2-3 weeks after discharge) was a deliberate choice. However, improvement in 
PHF may have occurred within that time span and may have influenced baseline scores of caregiver burden. As a result of these low baseline scores, a significant decrease in caregiver burden may have been difficult to achieve. For future studies we would recommend that specifically partners who experience high levels of caregiver burden are recruited in order to determine the effects of an intervention designed to alleviate this burden. Although the intervention indicated some effects on patient and partner health outcomes ${ }^{25}$, Martire and colleagues $^{23}$ describe that interventions that target only the family member and that are relationship-oriented, seem to be the most effective ones for decreasing caregiver burden. Therefore, our psycho-educational intervention in patient-partner dyads may not have been the optimal strategy to decrease feelings of burden in partners of patients with PHF. Furthermore, there may be other outcomes relevant to caregivers that the intervention was likely to influence, but that were not measured, e.g. caregiver competence, rewards of caregiving, satisfaction with caregiving, and management strategies of caregivers. ${ }^{30,31}$ Longitudinal data measuring caregiver burden over time is scarce. ${ }^{19,32}$ When looking at the evolvement of caregiver burden over time in both groups, it was found that the amount of performed tasks and perceived caregiver burden decreased, especially at 3 months after discharge. In another publication on the primary outcome of this pilot study, Ågren and colleagues $^{25}$ described that patients' perceived health and perceived control, and also partners' perceived health improved after cardiac surgery at 3 and 12 months (regardless of group). The improvement of the patients' perceived health may be associated with the decrease in caregiving tasks, such as assisting with personal care and emotional support.

Bivariate correlations at baseline and 12 months indicated consistent and significant associations between total burden scores, general strain, and feelings of isolation in general and practical support and motivational support in particular, with rho ranging between 0.31 and 0.60. Bivariate correlations at baseline were smaller or non-significant. This may be due 
to changes in the variability of scores and the decrease in overall levels of performed tasks and perceived burden over time. Although these associations seem stronger and more consistent compared to earlier studies ${ }^{19,28}$, performing caregiving tasks only partly explains perceived feelings of burden.

Several limitations of this study that may influenced the results have to be noted. As in most studies on caregiver burden, the overwhelming majority of participating partners were women, which limits the generalizability of the study. Also the unequal distribution of dyads between the two study groups may have been limitation. No information was available on pre-existing HF in patients. Therefore, some patients may have had pre-existing HF, which may have influenced the effect of the intervention in reducing the experience of burden in partners. The choice of instruments for measuring caregiver burden may have influenced the results. Since no caregiver burden instruments were available for partners of patients with postoperative HF, a HF specific measure for assessing performed caregiving tasks (the DOBI) was used, which may not have targeted caregiver tasks related to the surgical procedure. In general measurement of perceived caregiver burden is currently subject to discussion. Bastawrous ${ }^{33}$ stated that the concept of caregiver burden is often used, as an indicator of the caregiving experience. However, there is still much disagreement on the specific meaning and content of the term. Caregiver burden is most commonly investigated using quantitative measures, with little theoretical justification. As suggested by Bastawrous ${ }^{33}$ a mixed- method design integrating qualitative and quantitative approaches, may provide a more comprehensive understanding of the concept caregiver burden. Finally, the impact of interaction and mutual support within the dyad is currently acknowledged as an important factor influencing individual outcomes in partners as well as in patients, and should therefore be incorporated in future research on outcomes in caregivers and patients. ${ }^{34}$ 


\section{Conclusion}

A pilot study exploring the effects of a psycho-educational intervention in patients and their partners did not reveal significant effects with regard to reduced caregiver burden in partners. Caregiver burden scores seemed to decrease at 3 and 12 months after discharge in both groups. Considering the limitations of this small pilot study, the results should be interpreted with caution. Alleviating caregiver burden in partners may need a more intense or specific approach, but more large scale intervention studies are necessary to gain robust knowledge on effective intervention strategies to support partners and patients with post-operative heart failure. Intervention strategies specifically aimed at improving interaction and mutual support within the dyad may be worthwhile to explore. For future studies we would recommend recruiting partners who experience moderate to severe burden in order to be able to determine intervention effects on perceived caregiver burden. Furthermore, the use of mixed method designs is recommended in order to gain a more comprehensive understanding of the concept caregiver burden . 


\section{References}

1. Van Horn E. \& Tesh A. The effect of critical care hospitalization on family members: stress and responses. Dim Crit Care Nurs. 2000;19(4), 40-49.

2. Van Horn ER. Kautz, D. Promotion of family integrity in the acute care setting: a review of the literature. Dim Crit Care Nurs.2007; 26(3):101-117; quiz 8-9.

3. Agren S. Evangelista L. Davidson T. Strömberg A. The Influence of Chronic Heart Failure in Patient-Partner Dyads-A Comparative Study Addressing Issues of Health-Related Quality of Life. J Cardiovasc Nurs. 2011;26(1):65-73.

4. Rantanen A, Tarkka MT, Kaunonen M. et al, Health-related quality of life after coronary artery bypass grafting. J Adv Nurs. 2009; 65(9): 1926-36. doi: 10.1111/j.1365-2702.2011.03775.x.

5. Tully PJ, Baker RA, Turnbull D, Winefield H. The role of depression and anxiety symptoms in hospital readmissions after cardiac surgery. J Behav Med. 2008;31(4): 281-90. doi: 10.1007/s10865-008-9153-8.

6. Agren S, Frisman G, Berg S, Svedjeholm R, Stromberg A. Addressing spouses' unique needs after cardiac surgery when recovery is complicated by heart failure. Heart Lung. 2009;38(4): 284-291.doi: 10.1016/j.hrtlng.2008.10.002. Epub 2009 Jan 24.

7. McMurray JJ, Adamopoulos S, Anker SD. et al. ESC Guidelines for the diagnosis and treatment of acute and chronic heartfailure 2012: The Task Force for the Diagnosis and Treatment of Acute andChronic Heart Failure 2012 of the European Society of Cardiology. Developed in collaboration with the Heart Failure Association (HFA) of the ESC. Eur J Heart Fail. 2012; 14(8): 803-869.

8. Vanky FB, Hakanson E, Svedjeholm R. Long-term consequences of postoperative heart failure after surgery for aortic stenosis compared with 
coronary surgery. Ann Thorac Surg. 2007; 83(6): 2036-2043.

9. Vanky FB, Hakanson E, Tamas E, Svedjeholm R. Risk factors for postoperative heart failure in patients operated on for aortic stenosis. Ann Thorac Surg. 2006; 81(4): 1297-1304.

10. Holm, J.H.R., Vanky, F., Svedjeholm, R. Mixed venous oxygen saturation is a prognostic marker after surgery for aortic stenosis. Acta Anaesthesiol Scand. 2010; 54(5): 589-595. doi: 10.1093/bja/aer166. Epub 2011 Jun 16.

11. Chung ML, Moser DK, Lennie TA, Frasier SK. Perceived social support predicted quality of life in patients with heart failure, but the effect is mediated by depressive symptoms. Qual Life Res. 2013;22(7): 1555-1563. doi: 10.1007/s11136-012-0294-4. Epub 2012 Oct 18.

12. Årestedt K, Saveman BI, Johansson P, Blomqvist K. Social support and its association with health-related quality of life among older patients with chronic heart failure. Eur J Cardiovasc Nurs. 2013;12(1): 69-77. doi: 10.1177/1474515111432997. Epub 2012 Mar 28.

13. Gallagher R, Luttik ML, Jaarsma T. Social support and self-care in heart failure. J Cardiovasc Nurs. 2011;26(6): 439-45. doi: 10.1097/JCN.0b013e31820984e1.

14. Wu JR, Moser DK, Chung ML, Lennie TA. (2008). Predictors of medication adherence using a multidimensional adherence model in patients with heart failure. J Card Fail. 2008;14(7): 603-614. doi: 10.1016/j.cardfail.2008.02.011.

15. Schulz R, Beach SR. Caregiving as a risk factor for mortality: the Caregiver Health Effects Study. JAMA. 1999;282(23): 2215-2219.

16. Christakis N, Allison P. Mortality after the hospitalization of a spouse. $N$ Eng $J$ Med. 2006;354(7): 719-730.

17. Montgomery RV, Stull DE, Borgatta EF. Measurement and the analysis of burden. Res Aging, 1985;7(1), 137-152. 
18. Luttik ML, Jaarsma T, Tijssen JG, van Veldhuisen DJ, Sanderman R. The objective burden in partners of heart failure patients: development and initial validation of the Dutch Objective Burden Inventory. Eur J CardiovasC Nurs. 2008;7(1): 3-9.

19. Luttik ML, Jaarsma, T, Veeger N, et al. Caregiver burden in partners of Heart Failure patients: limited influence of disease severity. Eur J Heart Fail. 2007; 9(6-7): 695-701..

20. Given CW, Given B, Stommel M, et al. The caregiver reaction assessment (CRA) for caregivers to persons with chronic physical and mental impairments. Res Nurs Health, 1992;15(4): 271-283.

21. Hughes TB, Black BS, Albert M, Gitlin LN, Johnson DM, Lyketsos CG, Samus QM. Correlates of objective and subjective measures of caregiver burden among dementia caregivers: influence of unmet patient and caregiver dementia-related care needs. Int Psychogeriatr. 2014 Nov;26(11):1875-83. doi:

10.1017/S1041610214001240. Epub 2014 Aug 8.

22. Campbell TL. The Effectiveness of family interventions for physical disorders. $J$ Mar Fam Ther. 2003;29(2):263-281.

23. Martire LM, Martire LM, Lustig AP, Schulz R, Miller GE, Helgeson VS. Is it beneficial to involve a family member? A meta-analysis of psychosocial interventions for chronic illness. Health Psychol. 2004 Nov;23(6):599-611.

24. Hartmann M, Bäzner E, Wild B, Eisler I, Herzorg W. Effects of interventions involvingthe family in the treatment of adult patients with chronic physical diseases; a meta-analysis. Psychother Psychosom. 2010;79:136-148.

25. Agren S, Berg S, Svedjeholm R, Strömberg A. Psychoeducational support to post cardiac surgery heart failure patients and their partners - a 
randomised pilot study Intensive \& Critical Care Nursing. Intensive Crit Care Nurs. $2015 ; 31: 10-18$

26. Julious S.A. Sample size of 12 per group rule of thumb for a pilot study. Pharmaceutical Statistics, 2005;4:287-91.

27. Elmstahl S, Malmberg B, Annerstedt L. Caregiver's burden of patients 3 years after stroke assessed by a novel caregiver burden scale. Arch Phys Med Rehabil. 1996;77(2): 177-182.

28. Makdessi A, Harkness K, Luttik ML, McKelvie RS. The Dutch Objective Burden Inventory: validity and reliability in a Canadian population of caregivers for people with heart failure. Eur J Cardiovasc Nurs, 2011;10(4): 234-40. doi: 10.1016/j.ejcnurse.2010.08.006. Epub 2010 Oct 2.

29. Agren S, Evangelista LS, Hjelm C, Stromberg A. Dyads affected by chronic heart failure: a randomized study evaluating effects of education and psychosocial support to patients with heart failure and their partners. J Card Fail. 2012;18(5): 359-366. doi: 10.1016/j.cardfail.2012.01.014. Epub 2012 Mar 2.

30. Archbold PG, Stewart BJ, Miller LL, et al. The PREP system of nursing interventions: a pilot test with families caring for older members. Preparedness (PR), enrichment (E) and predictability (P). Res Nurs Health.1995;18(1): 3-16.

31. McKee K, Spazzafumo L, Nolan M, Wojszel B, Lamura G, Bien B. Components of the difficulties, satisfactions and management strategies of carers of older people: principal component analysis of CADI-CASI-CAMI. Aging Ment Health. 2009; 13(2): 255-64. doi: 10.1080/13607860802342219. doi: $10.1080 / 13607860802342219$. 
32. Pressler SJ, Gradus-Pizlo I, Chubinski SD, Smith G, Wheeler S, Sloan R, et al. Family caregivers of patients with heart failure: a longitudinal study. $J$ Cardiovasc Nurs. 2013;28(5): 417-28. doi: 10.1097/JCN.0b013e3182563877.

33. Bastawrous M. Caregiver burden - A critical discussion. Int J Nurs Stud 2013;50(3): 431-441. doi: 10.1016/j.ijnurstu.2012.10.005. Epub 2012 Nov 3.

34. Chung ML1, Moser DK, Lennie TA, Rayens MK The effects of depressive symptoms and anxiety on quality of life in patients with heart failure and their spouses: testing dyadic dynamics using Actor-Partner Interdependence Model. J Psychosom Res. 2009;67(1): 29-35. 
Table 1 . Study population

\begin{tabular}{|c|c|c|c|c|c|c|}
\hline & \multicolumn{2}{|c|}{ Patients } & & \multicolumn{2}{|c|}{ Partners } & \\
\hline & $\begin{array}{l}\text { Control } \\
(\mathrm{n}=17)\end{array}$ & $\begin{array}{c}\text { Experimental } \\
(n=25)\end{array}$ & $\begin{array}{c}p- \\
\text { value }\end{array}$ & $\begin{array}{l}\text { Control } \\
(\mathrm{n}=17)\end{array}$ & $\begin{array}{c}\text { Experimental } \\
(n=25)\end{array}$ & $\begin{array}{c}p- \\
\text { value }\end{array}$ \\
\hline Female $(\mathrm{n} / \%)$ & $1(6 \%)$ & $4(16 \%)$ & $0.63^{1}$ & $16(94 \%)$ & $21(84 \%)$ & $0.63^{1}$ \\
\hline Age (mean/standard deviation) & $69(8)$ & $70(9)$ & $0.69^{2}$ & $66(8)$ & $67(7)$ & $0.70^{2}$ \\
\hline $\begin{array}{l}\text { Co-morbidities }(\mathrm{n} / \%) \\
\text { Stroke } \\
\text { Diabetes } \\
\text { COPD }\end{array}$ & $\begin{array}{c}5(29 \%) \\
2(12 \%) \\
1(6 \%)\end{array}$ & $\begin{array}{c}6(24 \%) \\
4(16 \%) \\
-\end{array}$ & $0.75^{1}$ & & & \\
\hline $\begin{array}{l}\text { Education }(\mathrm{n} / \%) \\
\text { Primary school } \\
\text { Elementary school } \\
\text { Upper secondary school } \\
\text { University }\end{array}$ & $\begin{array}{c}6(35 \%) \\
8(47 \%) \\
1(6 \%) \\
2(12 \%)\end{array}$ & $\begin{array}{c}2(8 \%) \\
11(44 \%) \\
6(24 \%)^{*} \\
6(24 \%)^{*}\end{array}$ & $0.02^{3}$ & $\begin{array}{c}5(29 \%) \\
9(53 \%) \\
2(12 \%) \\
1(6 \%)\end{array}$ & $\begin{array}{l}3(12 \%) \\
6(24 \%) \\
8(32 \%)^{*} \\
8(32 \%)^{*}\end{array}$ & $0.01^{3}$ \\
\hline $\begin{array}{l}\text { Work }(\mathrm{n} / \%) \\
\text { Employed } \\
\text { Sickleave/Sickpension } \\
\text { Retired } \\
\text { Other }\end{array}$ & $\begin{array}{c}3(18 \%) \\
4(24 \%) \\
10(59 \%) \\
-\end{array}$ & $\begin{array}{c}4(16 \%) \\
4(16 \%) \\
17(68 \%) \\
-\end{array}$ & $0.56^{3}$ & $\begin{array}{l}6(35 \%) \\
9(53 \%) \\
2(12 \%)\end{array}$ & $\begin{array}{c}7(28 \%) \\
3(12 \%) \\
13(52 \%) \\
2(8 \%)\end{array}$ & $0.76^{3}$ \\
\hline
\end{tabular}


Table 2. CBS and DOBI mean (standard deviation) scores and significance levels for tested differences between intervention and control group

\begin{tabular}{|c|c|c|c|c|c|c|c|c|c|}
\hline & \multicolumn{2}{|c|}{ Baseline } & $p$-value* & \multicolumn{2}{|c|}{3 months } & p-valuel* & \multicolumn{2}{|c|}{12 months } & $p$-value* \\
\hline \multicolumn{10}{|c|}{$\begin{array}{l}\text { Subjective Burden (CBS) (range } \\
1-4)\end{array}$} \\
\hline Total score & $1.7 \pm 0.60$ & $1.7 \pm 0.57$ & 076 & $1.6 \pm 0.57$ & $1.4 \pm 0.55$ & 0.51 & $1.7 \pm 0.69$ & $1.5 \pm 0.44$ & 0.65 \\
\hline General strain & $1.8 \pm 0.68$ & $1.8 \pm 0.61$ & 0.84 & $1.6 \pm 0.67$ & $1.5 \pm 0.59$ & 0.71 & $1.7 \pm 0.78$ & $1.5 \pm 0.54$ & 0.62 \\
\hline Isolation & $1.7 \pm 0.78$ & $1.8 \pm 0.76$ & 0.60 & $1.5 \pm 0.50$ & $1.6 \pm 0.82$ & 0.90 & $1.8 \pm 0.71$ & $1.5 \pm 0.62$ & 0.32 \\
\hline Emotional involvement & $1.9 \pm 0.88$ & $1.7 \pm 0.76$ & 0.43 & $1.9 \pm 0.97$ & $1.5 \pm 0.83$ & 0.24 & $1.9 \pm 0.91$ & $1.7 \pm 0.67$ & 0.54 \\
\hline Environment & $1.5 \pm 0.63$ & $1.6 \pm 0.70$ & 0.83 & $1.3 \pm 0.42$ & $1.2 \pm 0.31$ & 0.76 & $1.5 \pm 0.42$ & $1.3 \pm 0.30$ & 0.30 \\
\hline \multicolumn{10}{|c|}{$\begin{array}{l}\text { Objective burden (DOBI) (range } \\
1-3 \text { ) }\end{array}$} \\
\hline Motivational support & $1.4 \pm 0.51$ & $1.3 \pm 0.33$ & 0.99 & $1.3 \pm 0.52$ & $1.2 \pm 0.19$ & 0.48 & $1.3 \pm 0.30$ & $1.2 \pm 0.24$ & 0.47 \\
\hline Emotional support & $2.0 \pm 0.53$ & $2.0 \pm 0.67$ & 0.90 & $1.7 \pm 0.53$ & $1.5 \pm 0.42$ & 0.40 & $1.9 \pm 0.61$ & $1.5 \pm 0.48$ & 0.08 \\
\hline
\end{tabular}

*Mann Whitney U test 
Table 3. Difference scores for the DOBI and CBS subscales per group (mean(standard deviation)) and p-values for tested differences in mean difference scores between groups

\begin{tabular}{|c|c|c|c|c|c|c|c|}
\hline \multirow{2}{*}{\multicolumn{2}{|c|}{ Difference variables per group }} & \multicolumn{3}{|c|}{ Baseline- 3 months } & \multicolumn{3}{|c|}{ Baseline -12 months } \\
\hline & & $\mathrm{N}$ & Mean (sd) & $p$-value* & $\mathrm{N}$ & Mean (sd) & p-valuel* \\
\hline \multirow[t]{2}{*}{ DOBI personal care } & Control group & 14 & $0.11(0.17)$ & & 13 & $0.18(0.31)$ & \\
\hline & Intervention group & 19 & $0.24(0.37)$ & 0.36 & 19 & $0.28(0.37)$ & 0.30 \\
\hline \multirow[t]{2}{*}{ DOBI emotional support } & Control group & 14 & $0.23(0.38)$ & & 13 & $0.08(0.46)$ & \\
\hline & Intervention group & 19 & $0.42(0.57)$ & 0.42 & 19 & $0.53(0.72)$ & 0.06 \\
\hline \multirow[t]{2}{*}{ DOBI motivational support } & Control group & 14 & $0.12(0.30)$ & & 13 & $0.11(0.46)$ & \\
\hline & Intervention group & 19 & $0.08(0.20)$ & 0.93 & 19 & $0.07(0.34)$ & 0.85 \\
\hline \multirow[t]{2}{*}{ DOBI practical support } & Control group & 14 & $0.19(0.32)$ & & 13 & $-0.01(0.44)$ & \\
\hline & Intervention group & 19 & $0.16(0.32)$ & 0.53 & 19 & $0.07(0.32)$ & 0.68 \\
\hline \multirow[t]{2}{*}{ CBS total } & Control group & 14 & $0.19(0.46)$ & & 13 & $0.07(0.32)$ & \\
\hline & Intervention group & 19 & $0.15(0.43)$ & 0.99 & 18 & $0.10(0.53)$ & 0.86 \\
\hline \multirow{2}{*}{ CBS general strain } & Control group & 14 & $0.17(0.62)$ & & 13 & $0.10(0.52)$ & \\
\hline & Intervention group & 19 & $0.17(0.49)$ & 0.99 & 19 & $0.20(0.67)$ & 0.57 \\
\hline \multirow{2}{*}{ CBS isolation } & Control group & 14 & $0.21(0.63)$ & & 12 & $0.00(0.55)$ & \\
\hline & Intervention group & 19 & $0.09(0.61)$ & 0.73 & 17 & $0.06(0.68)$ & 0.81 \\
\hline \multirow[t]{2}{*}{ CBS disappointment } & Control group & 14 & $0.24(0.50)$ & & 13 & $0.11(0.41)$ & \\
\hline & Intervention group & 19 & $0.13(0.49)$ & 0.65 & 19 & $0.08(0.66)$ & 0.82 \\
\hline \multirow[t]{2}{*}{ CBS emotional involvement } & Control group & 14 & $0.12(0.59)$ & & 13 & $0.15(0.59)$ & \\
\hline & Intervention group & 19 & $0.17(0.67)$ & 0.38 & 19 & $-0.10(0.60)$ & 0.54 \\
\hline \multirow{2}{*}{ CBS environmental } & Control group & 14 & $0.19(0.69)$ & & 13 & $-0.05(0.57)$ & \\
\hline & Intervention group & 19 & $0.33(0.68)$ & 0.68 & 19 & $0.24(0.60)$ & 0.05 \\
\hline
\end{tabular}

\footnotetext{
* Mann Whitney U test
} 